\title{
Measuring the Frequency Response of the Honeybee Thorax
}

\section{Author: Mark A. Jankauski}

[preface to the citation, regular font size, sentence case This phrasing may change as per publisher request please preference use of their language: "This is a [postprint/preprint/etc] of an article that originally appeared in [journal (linked)] on [date]". The final version can be found at [DOI].

[If there is a citation, include it on a line by itself, if grey literature, include the full date of this version (July 4, 2014). include a doi if possible] 


\title{
Measuring the Frequency Response of the Honeybee Thorax
}

\author{
Mark A. Jankauski \\ 220 Roberts Hall, Bozeman MT 59717 \\ E-mail: mark.jankauski@montana.edu \\ Date Submitted: December 2019 \\ Date Revised: February 2020
}

\begin{abstract}
Insects with asynchronous flight muscles are believed to flap at the effective fundamental frequency of their thorax-wing system. Flapping in this manner leverages the natural elasticity of the thorax to reduce the energetic requirements of flight. However, to the best of our knowledge, the fundamental frequency of the insect wing-muscle-thorax system has not been measured. Here, we measure the linear frequency response function (FRF) of honeybee Apis mellifera thoraxes about their equilibrium state in order to determine their fundamental frequencies. FRFs relate the input force to output acceleration at the insect tergum and are acquired via a mechanical vibration shaker assembly. When compressed $50 \mu \mathrm{m}$, the thorax fundamental frequency averaged across all subjects was about $50 \%$ higher than reported wingbeat frequencies. We suspect that the measured fundamental frequencies are higher in the experiment than during flight due to boundary conditions and posthumous muscle stiffening. Next, we compress the thorax between 100 $300 \mu m$ in $50 \mu m$ intervals to assess the sensitivity of the fundamental frequency to geometric modifications. For all specimens considered, the thorax fundamental frequency increased nearly monotonically with respect to level of compression. This implies that the thorax behaves as a nonlinear hardening spring when subject to large displacements, which we confirmed via static force-displacement testing. While there is little evidence that insects utilize this non-linearity during flight, the hardening characteristic may be emulated by small resonant-type flapping wing micro air vehicles to increase flapping frequency bandwidth. Overall, methods established through this work provide a foundation for further dynamical studies on insect thoraxes moving forward.
\end{abstract}

Keywords: Flapping wing flight, insect thorax, resonance, frequency response function, asynchronous muscle, Honeybee Apis mellifera, fundamental frequency

\section{Introduction}

Insects are remarkable fliers. They are capable of hovering [1], performing aggressive aerial maneuvers [2] and landing on surfaces in various orientations [3]. Given these capabilities, flying insects often serve as inspiration for miniature flapping wing micro air vehicles (FWMAVs) [4-6]. Insects have guided the design of FWMAV wings [7], 
actuators [8] and sensing modalities [9, 10]. While early designs have shown potential, FWMAVs continue to face technical challenges that must be overcome before they are suitable for widespread implementation. Among these challenges is improving vehicle energy efficiency to enable autonomous flight. Presently, many FWMAVs are tethered and require off-board sources to provide power [11]. A comprehensive understanding of the insect "drivetrain", the insect thorax in particular, may guide the design of energy efficient FWMAVs moving forward.

The thorax is the central component of the insect flight drivetrain (Fig. 1). It is an approximately ellipsoidal structure that contains two large sets of muscles called the dorsal-ventral (DVMs) and dorsal-longitudinal muscles (DLMs) [12]. As these muscles contract, they deform the thorax which indirectly causes the insect's wings to flap via an elaborate linkage mechanism called the wing hinge [13]. The insect may engage fine steering muscles in the hinge to maneuver [12]. Many insects leverage indirect wing actuation to flap [14], which in some cases is believed to reduce the power requirements of flight [15].

In addition to indirect actuation, many insects utilize a unique type of "asynchronous" flight muscle to reduce the energetic costs of locomotion [16]. Unlike synchronous muscles, there is no one-to-one ratio between muscle stimulation and mechanical contraction in asynchronous muscles - instead, they fire only once to initiate several wing beats. The dynamic properties of the thorax, rather than periodic muscle signaling, cause the muscle to repeatably contract in a process called stretch activation [14]. Interestingly, this muscle type has evolved independently numerous times along different branches of the insect phylogenetic tree, and researchers estimate the DVM and DLM flight muscles are asynchronous in approximately $75 \%$ of flying insects [16].

Much of the seminal research on asynchronous muscles was conducted by JWS Pringle in the 1950's and 1960's [17-20]. Pringle first observed that flight muscle length oscillations were higher and independent in frequency compared to neuron firing rate in the blowfly Calliphora erythrocephala [14]. Later, Pringle and colleagues attached a cyber-physical system with simulated mass, stiffness and damping to the asynchronous flight muscle of the Indian rhinoceros beetle Oryctes rhinoceros [18]. They found that the insect's muscle contraction frequency was equivalent to the fundamental frequency of the cyber-physical system to which it was attached. This suggested that the insect thorax-wing assembly, of which the flight muscles stiffen nontrivially, must have an effective resonant frequency equivalent to the insect's wingbeat frequency to support asynchronous muscle function. However, some insects vary their wingbeat frequency during flight $[21,22]$, whereas linear mechanical systems typically have fixed resonant frequencies. To explain this, researchers suggested that steering muscles could be used to stiffen the thorax $[23,24]$. Stiffening would increase the resonant frequency of the thorax, and as a result the insect's wingbeat frequency. 


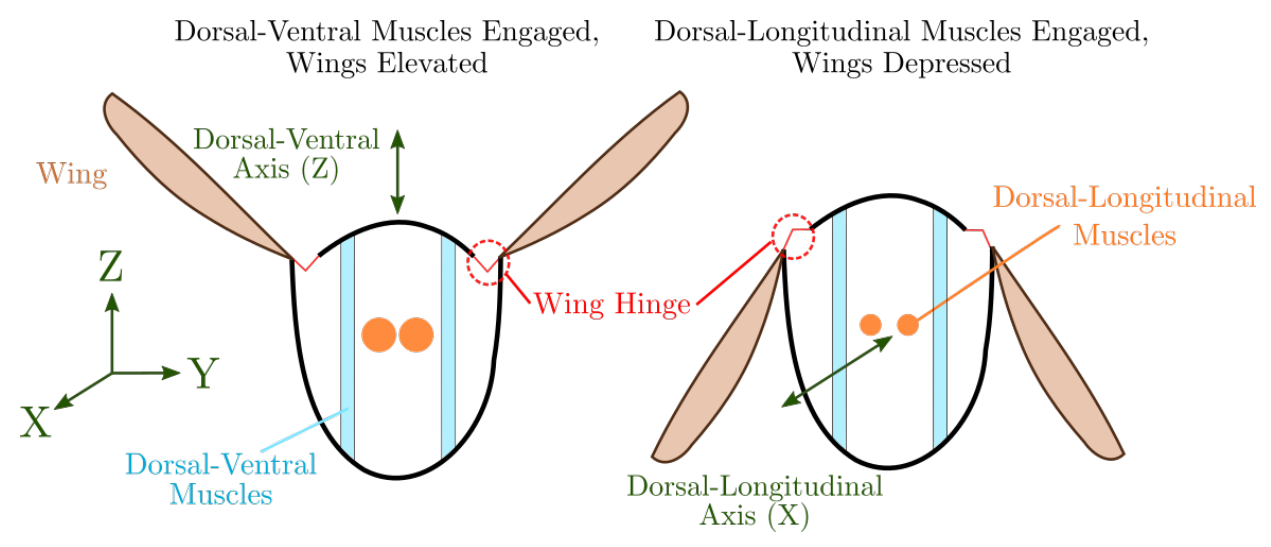

Figure 1: Simplified schematic of a thorax cross section for insects using indirect actuation. Dorsal-ventral muscle contractions cause the wings to elevate (upstroke) whereas dorsal-longitudinal muscle contractions cause the wing to depress (downstroke) [25].

\subsection{Scope of Present Research}

Despite indirect evidence that insect's flap at the fundamental frequency of their thorax, to the best of our knowledge the frequency response function (FRF) of the thorax has never been measured. Frequency response analysis, in the mechanical sense, involves exciting a structure over a wide frequency range with a known input signal and measuring the structure's response. The ratio between input and output in the frequency domain is the frequency response function (FRF). Spectral peaks in the FRF typically correspond to the structure's natural frequencies.

Given the motivation, the goals of the present work are to (1) determine the thorax FRF and to identify its fundamental frequency, and (2) assess if geometric modifications to the thorax achieved via compression cause its natural frequencies to change. For all experimental studies we use the honeybee Apis mellifera, which has asynchronous flight muscles. The first objective will, within the limitations of the experimental approach, indicate if honeybees' flap in proximity to the fundamental frequency of their thorax. To be clear, we refer to the first natural frequency of the thorax as its fundamental frequency. Though the thorax fundamental frequency may be influenced by the insect's wings, flight muscles and potentially abdomen and head, we generally refer to the system's aggregate fundamental frequency as the thorax fundamental frequency for brevity. The second objective will probe the sensitivity of the thorax's natural frequency to thorax shape. This work is restricted to describe only the small amplitude linear behavior of the thorax about varying equilibrium states. Nonlinear behavior in the dynamic sense is not considered.

We acknowledge that there are limitations to the experimental methods used in this manuscript which may lead to discrepancies between the measured thorax fundamental frequency the fundamental frequency of the thorax in-vivo. Some of these limitations include experimental boundary conditions, posthumous muscle effects, and lack of 


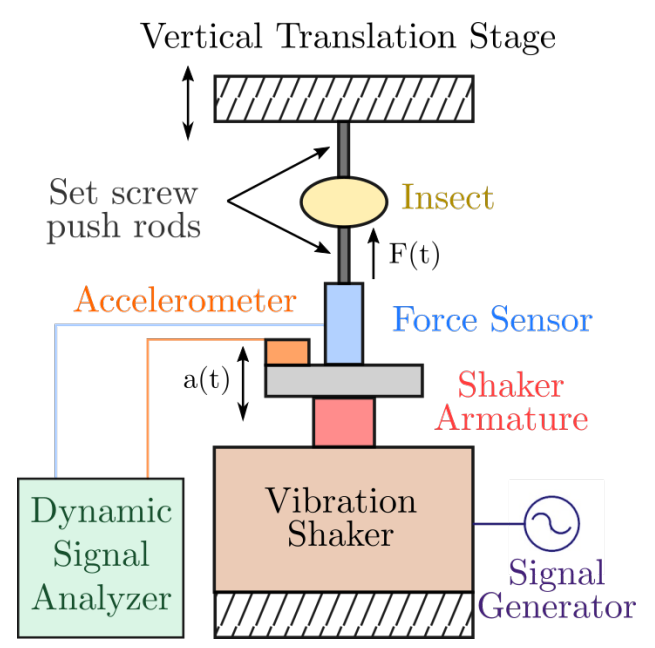

(a) Schematic of shaker assembly.

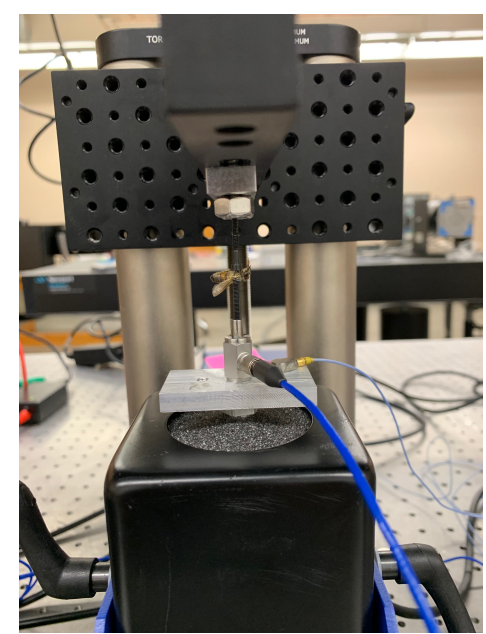

(b) Shaker assembly with honeybee clamped between push rods.

Figure 2: Experimental assembly used for measuring the insect thorax FRF.

steering muscle activity. Each of these potential sources of error is described within the manuscript where appropriate. Thus, all presented results must be viewed within the context of the experimental methods employed. Despite experimental limitations, this research provides insights into the honeybee thorax dynamics that are unavailable via other measurement techniques. The fundamental contribution of this work is therefore the novel method employed to measure the thorax FRF, which may be modified and extended to answer several open questions regarding thorax dynamics.

The remainder of this paper is organized as follows. We first detail the custom experimental setup used for dynamic testing as well as insect specimen collection and preparation. We then analyze FRF data and discuss the dynamic behavior of the thorax. Based upon these findings, we conduct a simple static analysis on the honeybee thorax. We conclude by examining the implications of this research.

\section{Methods}

We constructed a custom shaker assembly that allows us to excite an insect thorax about its dorsoventral axis (Fig. 2). Using this assembly, we determine the FRF $G(j \omega)$ that relates the force $F(j \omega)$ applied at a point on the ventral surface of the thorax to the acceleration $A(j \omega)$ at the same point, where $\omega$ denotes excitation frequency and $j$ denotes the imaginary unit. We define force as the input and acceleration as the output such that $G(j \omega)=\frac{A(j \omega)}{F(j \omega)}$. A mechanical idealization of the system is shown in Fig. 3 . We are able to determine the thorax's fundamental frequency via peaks in the FRF magnitude. 


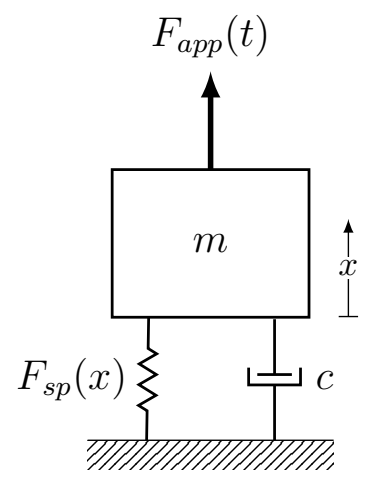

Figure 3: Mechanical representation of FRF experiment. $F_{a p p}$ denotes the force applied by the shaker, $x$ denotes thorax displacement, $F_{s p}(x)$ denotes the restorative force from the thorax muscles and exoskeleton, $c$ denotes a damping coefficient and $m$ is the effective mass of the system which includes the inertia of the thorax, wings and potentially the insect's head and abdomen.

\subsection{Dynamic Experimental Setup}

We use an electrodynamic vibration shaker (The Modal Shop, 2007E) rated at $31 \mathrm{~N}$ and powered by an external amplifier (The Modal Shop, 2100E21-100) to excite the thorax. A high-sensitivity piezoelectric force sensor (PCB Piezotronics, 209C11) threads directly into the shaker's insert. Then, a piezoelectric accelerometer (PCB Piezotronics, 352A21) is mounted via wax to a thick aluminum plate between the force sensor and shaker. The force sensor and accelerometer measure the input and output respectively, and are both powered by a benchtop signal conditioner (PCB Piezotronics, 482C15). The aluminum plate and force sensor fundamental frequencies much higher than the range of excitation frequencies considered in this work, and consequently do not affect the thorax's dynamic response.

We then place a large, coarse vertical translation stage (Thorlabs, VAP10) with a vertically mounted optical breadboard behind the shaker assembly (Fig. 2b). To enable fine vertical adjustment, we mount an additional one-axis translation stage (Newport, UMR8.25 stage with BM17.25 micrometer, $1.0 \mu \mathrm{m}$ resolution) to the vertical breadboard. An L-bracket is cantilevered off the fine linear stage. We thread a small push rod (\#2 screws) into a metal mounting plate, and attach this mounting plate directly to the L-bracket. We then thread a second \#2 screw directly into the force cell that is attached to the shaker. The two screws are colinear such that when the vertical stage is lowered, the insect thorax is compressed between them. We use \#2 set screws to minimize the contact area between the push rod and the thorax. Insects are mounted upside down such that their tergums are aligned with the force sensor. When mounting an insect, we use small beads of glue applied via a needle point to secure the dorsal and ventral surfaces of the thorax to their respective push rods. Once an insect is mounted, we lower the vertical stage until the insect's wings begin to articulate. 
We use a dynamic signal analyzer (Data Physics, ABACUS 901) both to record force and acceleration data as well as to generate excitation reference signals. The signal analyzer converts time-domain measurements to the frequency domain via a fast Fourier transform (FFT). We actuate the shaker with a "periodic chirp" from 10 - 1000 $\mathrm{Hz}$ over a $400 \mathrm{~ms}$ period with a signal width of $60 \%$. Displacement, as estimated by twice integrating the acceleration measurement, varies with excitation frequency but is typically restricted to less than $\pm 1 \mu \mathrm{m}$ to ensure that the thorax is excited in the linear range. Data is recorded at a rate of $2560 \mathrm{~Hz}$, resulting in $400 \mathrm{FFT}$ lines with a frequency resolution of $2.5 \mathrm{~Hz}$. We utilize a Hanning window during data collection and data is averaged over at least 50 periods to reduce noise. Through this procedure, we determine the FFT $G(j \omega)$ relating acceleration and force as measured at the insect tergum. We then identify the thorax's fundamental frequency by fitting $G(j \omega)$ via MATLAB's modal fitting tool with peak picking (PP) algorithm. In instances where the peak peaking algorithm failed to fit the FRF, we opted for a least-squares rational function (LSRF) fitting algorithm. In both cases a 10-sample moving mean filter was applied to the frequency domain data set to improve the accuracy of the fit.

Once data is collected for a level of thorax compression, we compress the thorax further using the fine linear stage and repeat the experiment. For the purposes of this work, we consider a compression range of 50 - $300 \mu \mathrm{m}$ in $50 \mu \mathrm{m}$ increments. We found that a minimal level of compression was required to get clean FRF measurements, and that approximately $300 \mu \mathrm{m}$ of dorsoventral compression was required to fully articulate the honeybee's wings (Fig. 4). While the angle of articulation is dependent on the state of the insect's wing hinge after sacrifice, the wing typically rotated 55-70 degrees across the honeybees tested. This rotation is referenced from a fixed transverse plane intersecting the insect's body. Honeybees flap with stroke amplitudes approximately 45 - 65 degrees [26], though we note the reference planes from which angles are measured vary from study to study. Furthermore, our static compression does not induce the same span-wise bending and chord-wise twisting most wings experience during dynamic flapping, which may further increase stroke angle [27]. Nonetheless, our values of maximum wing articulation are within proximity to those measured in flight, particularly given the experimental limitations. On the other hand, the compression needed to achieve such articulation is likely much higher than the thorax would experience in nature. Though we were unable to find thorax displacement measurements for the honeybee, we look to the stingless bee Melipona seminigra to provide an estimate.

Displacements of the stingless bee Melipona seminigra thorax were estimated at $20 \mu \mathrm{m}$ peak-to-peak during tethered flight and up to $50 \mu \mathrm{m}$ peak-to-peak during "annoyance buzzing" behavior [28]. One potential issue with using these estimates to inform our study, however, is that thorax velocity (from which displacement was estimated via integration) was measured at a single point on the thorax's dorsal surface with laser vibrometry. Ando and Kanzaki measured the dorsal surface of a hawkmoth Agrius convolvuli thorax using a high-speed profilometer found displacements varied significantly along the medial-lateral direction [29]. Thus, it is likely that thorax 


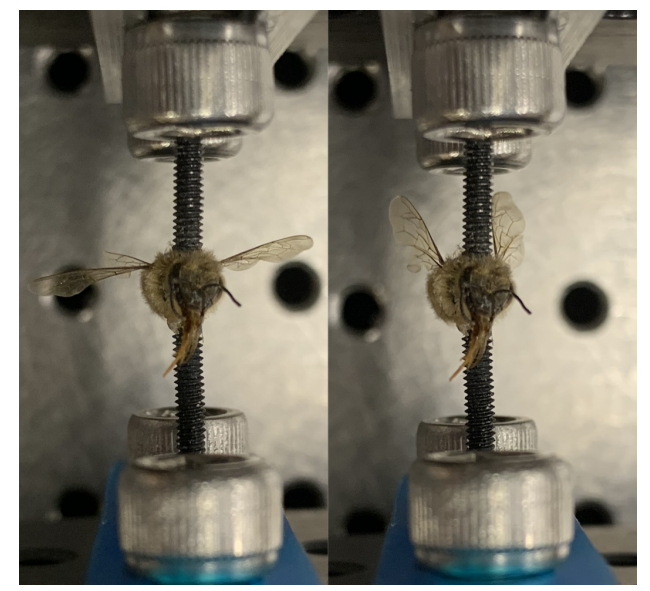

Figure 4: (Left) Uncompressed honeybee with wings in resting position and (Right) Honeybee with thorax compressed $300 \mu \mathrm{m}$ with wings articulated approximately $65^{\circ}$ measured with respect to a fixed transverse plane intersecting the insect's body.

displacements estimated via single point laser vibrometry are sensitive to the precise location of the measurement. Further, the push rods deform the thorax in a manner dissimilar to the flight muscles. In general, the dorsal-ventral flight muscles attach near the wing hinge, whereas the push rod compresses the thorax at a point along the medial line of the scutum. Depending on the material and geometric thorax stiffness at the medial line and closer to the wing hinge, it is possible the thorax must be compressed by the push rod a greater distance than the muscles would typically contract in real flight to cause maximum wing articulation. We maintain the 50 - $300 \mu \mathrm{m}$ range to ensure we capture relevant data both inside and potentially outside the anatomical range, with the understanding that induced deformations will deviate from natural deformations. We do not consider tensile loading in this study.

\subsection{Insect Collection and Preparation}

Honeybees were collected while foraging at Montana State University's (MSU) pollinator garden. All specimens were collected and transported to the laboratory immediately before experimentation and were housed in containers up to several hours. Insects were sacrificed in a sealed jar containing plaster saturated in ethyl acetate. We carefully removed legs from the ventral surface of the thorax in order to expose a smooth mounting surface for the push rods. The insect's wings were left intact. Following euthanization, experiments lasted maximally one hour (typically around twenty minutes) to minimize desiccation or other effects that may potentially affect the dynamic properties of the thorax. 
Table 1: Tabulated FRF data for all honeybee subjects $(n=7)$. The values in the gray colored boxes indicate thorax fundamental frequencies (in $\mathrm{Hz}$ ) for a subject and compression level. $\dagger$ indicates that the FRF was fit via a LSRF fitting function instead of a PP fitting function. We found that some thoraxes rotated during dynamic testing, thereby changing thorax alignment and distorting FRF data. These trials were discarded.

\begin{tabular}{|c|c|c|c|c|c|c|}
\hline \multirow[b]{2}{*}{ Subject } & \multicolumn{6}{|c|}{ Compression } \\
\hline & $50 \mu m$ & $100 \mu m$ & $150 \mu m$ & $200 \mu m$ & $250 \mu m$ & $300 \mu m$ \\
\hline 1 & 298.4 & 357.1 & 397.5 & 360.5 & 420.3 & 427.6 \\
\hline 2 & 421.6 & 433.7 & 456.7 & 475.1 & 483.6 & 496.0 \\
\hline 3 & 375.5 & 397.1 & 399.0 & 390.1 & 423.3 & $416.6^{\dagger}$ \\
\hline 4 & 411.5 & 419.9 & 442.2 & 448.9 & 445.0 & 454.6 \\
\hline 5 & 316.3 & 338.2 & 379.5 & 380.7 & 405.3 & 421.1 \\
\hline 6 & 356.1 & 414.4 & 432.3 & 415.0 & 409.6 & 421.0 \\
\hline 7 & 395.6 & 394.5 & 401.9 & 425.0 & 445.0 & 462.0 \\
\hline avg & 367.8 & 393.6 & 415.6 & 413.6 & 433.2 & 442.7 \\
\hline
\end{tabular}

\section{Results}

In this section, we use the experimental setup to investigate the linear dynamics of the insect thorax. We first compare measured thorax fundamental frequencies to reported values of wingbeat frequency. We then address the sensitivity of fundamental frequency with respect to compression. Finally, we conduct a static force-displacement test to identify the stiffness of the honeybee thorax.

\subsection{Thorax Fundamental Frequency}

All effective thorax fundamental frequencies shown in Tab. 1, and one indicative FRF for a honeybee thorax compressed $50 \mu m$ is shown in Fig. 5. These results suggest that the fundamental frequency of the honeybee thorax is in proximity to the insect's wingbeat frequency. At $50 \mu \mathrm{m}$ of compression, the average thorax fundamental frequency is 368 $\mathrm{Hz}$ for the honeybee. Previous literature indicates the wingbeat frequency is $220-250 \mathrm{~Hz}$ for the honeybee during flight [26]. While the average measured fundamental frequency is about $50 \%$ higher than the upper bound of the reported wingbeat frequency, there are several factors that may have caused the thorax frequencies to be higher during the experiment than during in-vivo flight. Potential sources of error are discussed here.

First, experimental boundary conditions may artificially stiffen the thorax, thereby increasing its fundamental frequency. During flight the thorax is free to oscillate, whereas in the experiment the dorsal and ventral surfaces are constrained by push rods (Fig. 2). Constraints imposed on a structure may increase its natural frequencies. To approximate the effect of boundary conditions, we develop a simple finite element 
Magnitude vs. Frequency

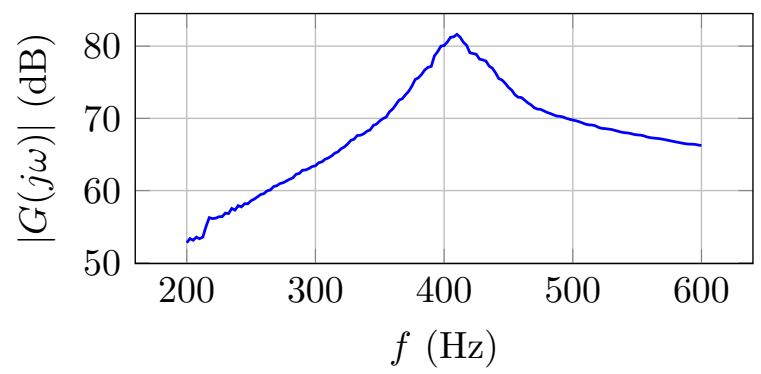

Phase vs. Frequency

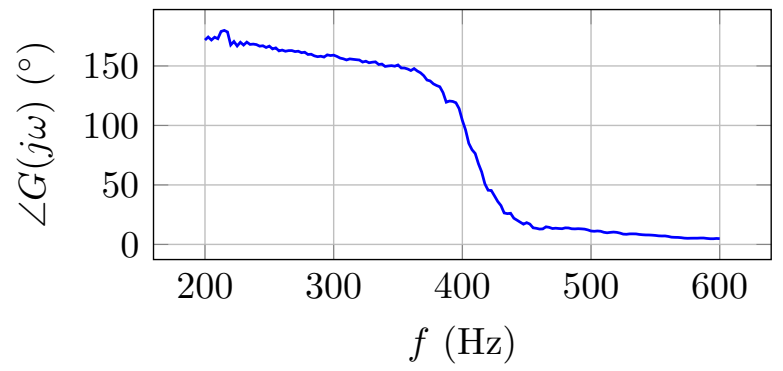

Figure 5: Frequency response function magnitude and phase of a honeybee thorax (subject 4) compressed $50 \mu \mathrm{m}$. The sharp magnitude peak and $180^{\circ}$ phase shift indicate a resonance at $411 \mathrm{~Hz}$. Data is unfiltered.

(FE) model of a honeybee thorax idealized as a circular hoop and calculate numerically its natural frequencies with and without constraints (Appendix A). The FE model is not intended to replicate the thorax, but provides an approximation of how boundary conditions affect its fundamental frequency. Comparing a free hoop to a hoop with boundary conditions similar to the experiment, we find that single point pin constraints at the top and bottom of the hoop increase its first natural frequency by $25 \%$. This percentage increases if larger constraint contact areas are considered. The boundary conditions imposed by the experiment may therefore stiffen the insect thorax and increase its fundamental frequency non-trivially.

Second, insect flight muscles may stiffen immediately following sacrifice due to rigor mortis. While we were unable to find data specific to honeybees or Hymenoptera, rigor mortis is expected to set in within minutes of death in Lepidoptera muscle [30]. Previous studies have shown that, for the asynchronous beetle flight muscle, the active muscle is approximately $40 \%$ stiffer than the passive muscle [16]. If rigor mortis were to cause a stiffening effect of similar magnitude, it may also contribute to the higher fundamental frequency measured in the experimental setting, where FRFs measured within one hour of specimen sacrifice. Unfortunately, it is difficult to reconcile this muscle effect in sacrificed insects. The muscles will eventually relax over time as rigor mortis fades, however the thorax will simultaneously desiccate. While the effect of desiccation to thorax stiffness has not been studied to our knowledge, desiccation in some insect wings has been accompanied by an increase in stiffness and natural frequency [31]. Moving forward, it is advisable to repeat the studies with anesthetized insects to minimize the influence of these posthumous muscle effects.

Lastly, it is possible that realistic dynamic coupling between the thorax and wing is not achieved in the experimental context. Hrncir showed that removal of one or both wings in tethered flying stingless bees led to an increase in thorax oscillation frequency [28]. At the same time, wing removal caused no increase in thorax oscillation frequency during annoyance buzzing, where the wings would typically be folded over the 
Fundamental Frequency vs. Compression

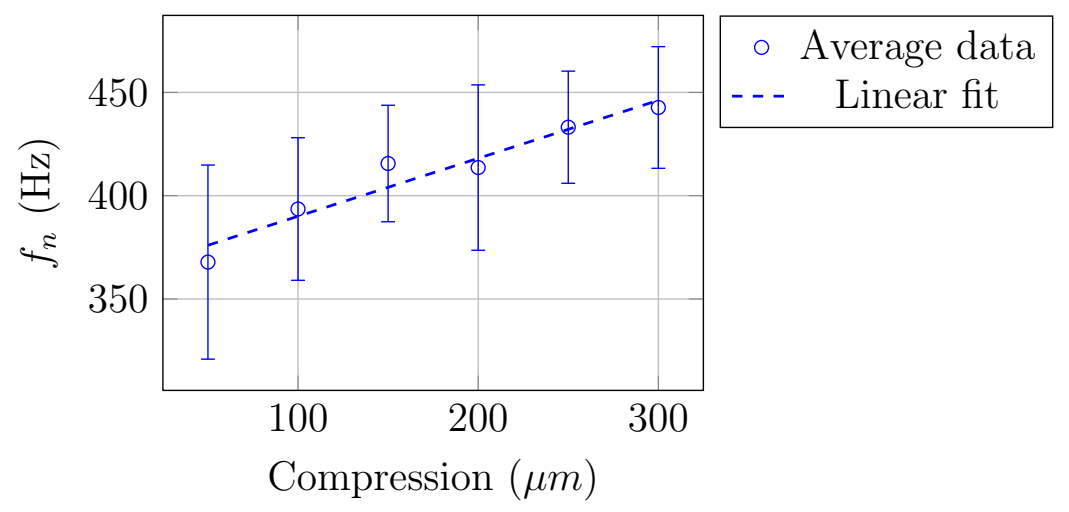

Figure 6: Average fundamental frequency, $f_{n}$, as a function of dorsal-ventral compression for honeybee specimens $(n=7)$. Bars show standard deviation.

insect's abdomen during this behavior. This suggests that the wing-thorax fundamental frequency is sensitive to wing orientation, where the wing reduces the fundamental frequency when extended such that its center of mass is cantilevered away from the insect body. In this extended orientation, added mass and aerodynamic drag (which is dependent on flapping amplitude) are also increased due to the larger exposed surface area; both fluid effects may also contribute to the reduction the wing-thorax fundamental frequency. While the wings were extended during our studies, and articulated with thorax compression, it is feasible that load transfer between the thorax and wing is sensitive to the precise configuration of the wing hinge as well as steering muscle activity. Moreover, at the low compression levels utilized during the experiment, it is unlikely that aerodynamic drag and the subsequent reduction in wing-thorax fundamental frequency is as appreciable. All of this suggests that the wing-thorax fundamental frequency may be higher in the experiment than during flight if the wing hinge is not engaged properly and operating at large amplitude.

Thus, there are several factors that may increase the thorax fundamental frequency during the experimental setting, though it is challenging to decouple their individual contributions. Additional research is required to address each factor independently. Nonetheless, the measurements taken here appear to support the idea that honeybees flap at the fundamental frequency of their thorax, particularly if the potential sources of error are considered.

\subsection{Sensitivity of Fundamental Frequency to Compression}

Motivated by the notion that insects are able to change wingbeat frequency, we now explore the effect of compression on thorax fundamental frequency. Note that while the insect's wings articulate as we compress the thorax during our experiment, there are potential mechanisms by which the thorax can deform without causing the wings to flap. This seems likely given that some of the steering muscles attach directly to the 
thoracic walls [32]. Therefore, although the changes in thorax fundamental frequency are accompanied by a change in mean stroke angle in our experiment, it is possible that the fundamental frequency can be modulated in real flight without significantly influencing flapping kinematics.

Interestingly, the thorax fundamental frequency tends to increase with compression for all specimens considered (Tab. 1). The average fundamental frequency as a function of compression level for the honeybee is shown in Fig. 6. With the exception of the modest drop in average fundamental frequency for the honeybee between $150 \mu \mathrm{m}$ and $200 \mu \mathrm{m}$, this trend occurs monotonically. This implies that the thorax's fundamental frequency is sensitive to its instantaneous shape, which may provide a simple mechanism for the insect to adjust wingbeat frequency during flight. Prior research suggests insects from the order Diptera modulate their wingbeat frequency by tensioning their thorax via pleurosternal steering muscles [12]. While our experiment does not emulate this tensioning mechanism, it does support the idea that thorax modifications can alter its dynamic response.

The ability to modulate the thorax's fundamental frequency is important for flight and other behaviors. Some Hymenoptera (although not the honeybee) perform a behavior called buzz pollination [33]. During buzz pollination, the bee will land on a flower, disengage its wings and excite its flight muscles at high frequency. The high frequency vibrations help dislodge the flower's pollen so that the bee can collect it more easily. Flight muscle oscillations during buzz pollination occur a much higher frequency than during flight, which implies the fundamental frequency of the thorax is higher during the buzz pollination behavior. The increase in thorax fundamental frequency may result from the bee disengaging its wings, since disengaging the wings reduces the effective inertia of the wing-thorax system.

However, some bees vary their buzz pollination frequency depending on the physical attributes of the flower they are collecting pollen from [34]. This suggests that the bee can adjust its thorax fundamental frequency even while its wings are disengaged. Further, King et al. showed that during buzz pollination, carpenter bees adjusted the mean position about which their thorax oscillates [35], which may indicate muscle tensioning. It is likely that both the reduction of inertia due to wing disengagement, as well as an increase in muscle (potentially steering muscle) tension, contribute to the high frequency oscillations observed during buzz pollination.

\subsection{Hardening Spring Behavior}

We observed that the honeybee thorax fundamental frequency increased with compression level. This suggests that the honeybee thorax behaves as a nonlinear hardening spring, where its stiffness increases with respect to its degree of deformation [36]. Gau et al. also observed this hardening behavior in the hawkmoth Manduca sexta thorax about its longitudinal axis during low frequency forcedisplacement measurements [15]. To further understand this behavior in the honeybee, 


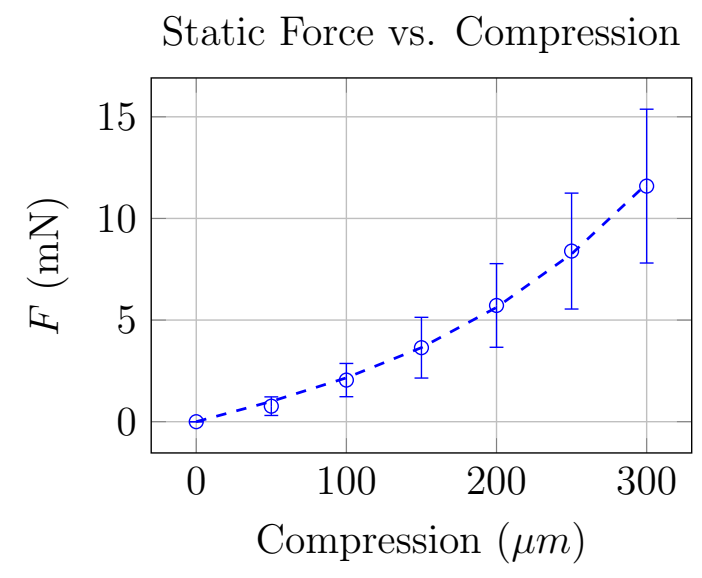

Figure 7: Average dorsal-ventral force-displacement curve of honeybee thorax $(\mathrm{n}=10)$ specimens. Bars show standard deviation.

we measure the static dorsal-ventral force-displacement curve its thorax $(n=10)$. The derivative of the curve with respect to displacement yields the local thorax stiffness.

We use the same general experimental set-up for static testing with two key differences: (1) the vibration shaker is replaced with a rigid support, and (2) the piezoelectric force sensor is replaced with a high-sensitivity foil-based load cell (Transducer Techniques, GSO-100). We use a National Instruments cDAQ to record force data. For the purposes of this work, we recorded the load immediately after the thorax was compressed to minimize the effect of hysteresis on measurements. We considered only increasing compression levels (loading) and did not record the unloading phase of the thorax. Results are shown in Fig. 7.

These results verify that the honeybee thorax behaves as a hardening spring. The slope of the force-displacement, which increases with respect to thorax compression, defines the thorax's local stiffness. We curve fit to average data from Fig. 7 to determine the thorax's elastic force $F_{s p}(x)$ as a function of compression $x$. Assuming a linear fit with an intercept of zero, the coefficient of determination $R^{2}=0.9802$. For a linearcubic fit of the form $F_{s p}(x)=a_{1} x^{3}+a_{2} x$ the fit improves to $R^{2}=0.9992$. We maintain the linear-cubic fit for subsequent analysis due to the higher coefficient of determination.

In general, the thorax's fundamental frequency will be proportional to its mass and stiffness. If we assume the mass is constant, thorax's fundamental frequency $f_{n}$ roughly scales with

$$
f_{n} \propto \frac{d F_{s p}}{d x}=\sqrt{3 a_{1} x^{2}+a_{2}}
$$

The above suggests that $f_{n}$ will increase linearly when $x$ is large. To assess this, we plot the average fundamental frequency versus compression from Tab. 1 for all insects as well as a linear fit of the data in Fig. 6. In general, the linear fit models the increase in $f_{n}$ fairly well $\left(R^{2}=0.934\right)$. Our findings substantiate the idea that the honeybee thorax behaves as a nonlinear hardening spring over the compression range considered, which 
we acknowledge may extend beyond the anatomical range of the thorax deformations.

The exact physical mechanism underlying the hardening nonlinearity is difficult to discern, though there are several factors that may contribute. Thorax geometry most almost certainly plays a role. Shallow arches on elastic supports, which could serve as an idealized structural model of the thorax, are known to behave as hardening springs under certain loading conditions [37]. Even so, a simplified monolithic structure like an arch or hoop may not adequately capture the geometric complexity of the honeybee thorax, which is comprised of distinct segments connected via membrane and suture [32]. Furthermore, since the experimental boundary conditions increased thorax stiffness during dynamic testing, it is plausible they have a similar effect during this static test.

In addition to geometric effects, the nonlinearity in force-displacement may also arise from thorax material properties. The thorax is made up primarily of chitin, resilin and other various proteins and lipids $[32,38,39]$. Chitin is typically rigid and stiff, whereas resilin is highly elastic to permit mobility and large deformation. Despite the known presence of these materials in the thorax, to the best of our knowledge their distributions have not been mapped in three dimensions. Further, functional gradation of these materials has not been studied extensively via nano-indentation or other micro-mechanical characterization techniques. These micro-scale characteristics may also affect the nonlinear macroscale response. Lastly, the passive flight muscles themselves may contribute to nonlinearity. Josephson and Ellington observed a nonlinear stress-length relationship in the bumblebee Bombus terrestris flight muscle for large strains [40]. Machin and Pringle reported a similar finding for the passive baslar muscle in lamellicorn beetles [18].

\section{Discussion}

In this work, we investigated the hypothesis that many insects flap at the fundamental frequency of their thorax. To assess this hypothesis, we identified the effective fundamental frequency of the honeybee thorax by characterizing its linear frequency response function. We found that, when compressed minimally, measured thorax fundamental frequencies were approximately 50-70\% higher than the insects' reported wingbeat frequency. However, the experimental assembly constrains the thorax in a manner that may significantly stiffen it, thereby increasing its fundamental frequency relative to the free boundary conditions that would be experienced during flight. Other factors, such as rigor mortis or inactive steering muscles, may also increase the thorax fundamental frequency measured experimentally. We believe that, if these errors are reconciled, the fundamental frequency of the thorax would indeed be equivalent to the insect's wingbeat frequency. The novel FRF methodology developed in this work establishes a foundation to address these experimental influences individually and provides a new technique to study insect thorax dynamic behavior.

Next, we compressed the thorax and measured its FRF to identify if the thorax's 
fundamental frequency was sensitive to changes in geometry. We found that the thorax's fundamental frequency increased with respect to compression, although the compression levels considered may exceed the anatomical range of muscle contraction in real insects. Nonetheless, this hardening behavior suggest the honeybee thorax may behave as a weakly nonlinear oscillator, which may explain the presence of superharmonics in previous recordings of insect thorax oscillations [28]. Further analysis of this research, including the energetic benefits of flapping at resonance and the potential nonlinear dynamics of the thorax, is discussed in the following sections.

\subsection{The Energetics of Flapping at the Thorax Fundamental Frequency}

Our results that flying insects likely do flap at the fundamental frequency of their thorax. Why is this beneficial? Consider the honeybee thorax FRF shown in Fig. 5. At the thorax's fundamental frequency, the FRF magnitude $|G(j \omega)|$ increases dramatically while the phase undergoes a $180^{\circ}$ shift. The peak of $|G(j \omega)|$ occurs due to a minimum in the force magnitude $\left|F_{a p p}(j \omega)\right|$, which implies less force is required to oscillate the thorax at this frequency. In general, the energy required to flap the wings is equivalent to the work $W$ done by the thoracic force, or

$$
W=\int_{C} F_{a p p} \cdot d S
$$

where $C$ is the trajectory of which the force acts and $d S$ is the differential distance. In general, the thorax trajectory $C$ is fixed because a certain amount of deformation is required to articulate the wings. Then, in order to reduce the energy required to flap the wings, the applied force must be lowered. This is achieved via flapping at resonance. In a sense, flapping in this manner maximizes the work done by conservative forces, namely the passive elastic forces of the thoracic exoskeleton and flight muscles. These forces are responsible for slowing the wing down or "braking". Absent thorax and muscle elasticity, energy would have to be invested both to accelerate and decelerate the wing. Therefore, insects may flap at the fundamental frequency of their thorax in order to reduce the energetic requirements of flight.

\subsection{Thorax as a Duffing Oscillator}

The experiments conducted here considered low amplitude excitation and were designed only to force the thorax in its linear range. However, the results of this research suggest that the thorax may behave as a weakly non-linear oscillator when excited at large amplitudes, albeit perhaps at larger amplitudes then are conventionally experienced in nature. Notably, the thorax behaves as a nonlinear hardening spring, which suggests that its stiffness and fundamental frequency increase with respect to a constant level of compression. The cubic stiffness nonlinearity is the hallmark of the Duffing oscillator [36]. With linear damping and external harmonic forcing, the equation of motion for the Duffing oscillator is

$$
\ddot{x}+\delta \dot{x}+\alpha x+\beta x^{3}=\gamma \cos (\omega t)
$$


where $\delta$ is the linear viscous damping coefficient, $\alpha$ is the linear stiffness coefficient, $\beta$ is the cubic stiffness coefficient, $\gamma$ is the excitation amplitude and $\omega$ is the harmonic forcing frequency. The behavior of the Duffing oscillator is well studied and may provide insight into the nonlinear mechanics of the insect thorax.

One notable feature of the Duffing oscillator is that, when subject to a forcing with periodicity of $\omega$, it responds both at $\omega$ and integer harmonics thereof. Hrncir et. al observed such harmonics when measuring the thorax during "annoyance buzzing" in bees [28]. Because thorax deformation also drives flapping, it is possible these harmonics affect flapping kinematics as well. Recordings of hawkmoth Manduca sexta wingbeat kinematics indicate the presence of higher-order harmonics of the flapping frequency in all three wing rotations [41]. Higher-order harmonics of the flapping frequency appear to play a significant role in wing deformation and the corresponding energetic and aerodynamic efficiency of flight [42-44].

A second essential feature of the Duffing oscillator is its amplitude dependence. Unlike linear oscillators, the frequency at which the Duffing oscillator exhibits the largest response depends on excitation amplitude. This diverges from linear oscillators where the fundamental frequency depends on only the properties of the structure and not the excitation amplitude. Though this would be an interesting mechanism to affect wingbeat frequency, there is little evidence that honeybees leverage amplitude dependence for this purpose. Many species of bees adjust their stroke amplitude to produce additional lift to climb or carry loads while maintaining their wingbeat frequency [45-47]. It is believed that these changes in wing kinematics stem primarily from the flight muscle. Aside from using steer muscles to brace the thorax, change of wingbeat frequency in bees is typically correlated to wing wear, which causes a reduction in effective inertia of the wing-thorax system [48], or changes in ambient temperature which affects muscle performance [49]. To our knowledge, there are no studies that definitively show a relationship between muscle strain amplitudes and wingbeat frequency.

Nonetheless, the amplitude dependent behavior of the Duffing oscillator may be desirable for FWMAVs to mimic. Many smaller FWMAVs rely on resonance between the coupled wing and actuator (often piezoelectric) to realize large stroke amplitudes [4]. While this is an effective way to amplify small actuator displacements, it permits only a narrow range of permissible flapping frequencies. Once the flapping frequency deviates significantly from the system's fundamental frequency, its response amplitude and wing rotation attenuate starkly. In contrast, leveraging an amplitude-dependent nonlinear system will increase the permissible range of flapping frequencies, because the system's fundamental frequency can be increased by strengthening actuator amplitude. This may provide an efficient mechanism to initiate energy efficient climbing flight, because both the stroke amplitude and wingbeat frequency increase simultaneously while the resonant behavior of the system is preserved. It is challenging to establish a general strategy to incorporate Duffing behavior into FWMAVs given their varied designs, however we believe the simplest way to do so without adding mass to the system is to leverage geometric nonlinearity. Shallow arches can exhibit both hardening or softening behavior 
based upon their initial configuration [50]. For a FWMAV that utilizes piezeoelectric actuation, one may incorporate an initial curvature into the actuator via boundary constraints in order to exploit this Duffing behavior. For larger FWMAVs with increased payload, the options for implementing non-linearity are greater, for example realizing hardening behavior via a simple magnetic spring.

\section{Acknowledgments}

This manuscript was supported in part by the National Science Foundation under award No. CBET-1855383. Any opinions, findings, and conclusions or recommendations expressed in this material are those of the author(s) and do not necessarily reflect the views of the National Science Foundation. The authors declare no conflict of interest regarding the publication of this article. We would like to thank Keith Fisher for retrieving the paper wasp nest, Dr. Andrew Mountcastle for his useful discussion on buzz pollination, and Data Physics Inc. for providing the dynamic signal analyzer.

\section{Appendix A - Simplified Finite Element Modeling}

We develop a simplified finite element model (Fig. 8) to estimate how experimentally boundary conditions affect the fundamental frequency of the insect thorax. The simple model is not intended to reproduce experimental findings, but instead provide insight into how the push rod constraints (Fig. 2) influence the thorax's dynamic response.

We idealize a transverse cross section of the thorax as a planar, uniform thin-walled hoop with a radius of $3 \mathrm{~mm}$. We model only half the hoop and assume symmetry about a vertical line bisecting the hoop. The hoop wall is solid and circular with a radius of $10 \mu \mathrm{m}$, density of $\rho=1000 \mathrm{~kg} / \mathrm{m}^{3}$ and Young's modulus $E=3 \mathrm{GPa}$. The modeled hoop section is discretized into 50 beam elements of equal lengths, which is a sufficient for convergence of the structure's first several natural frequencies.

We consider free-free boundary conditions, which is representative of free flight, and pinned-pinned boundary conditions, which is similar to those imposed during the experiment. For the free-free case, single points at the top and bottom of the circle are constrained such that they can only translate in the $y$ direction; $z$ axis rotation and $x$ translation are restricted. These are necessary boundary conditions when assuming the thorax is symmetric about $y$ axis. For the pinned-pinned case, the points at the top and

bottom of the circle are constrained to have no translation. For both cases, we conduct numerical modal analysis to identify the first two natural frequencies and corresponding mode shapes of the idealized thorax (Fig. 8). Note that the first natural frequency of the free-free model will be zero, which indicates rigid body translation of the structure in the $y$ direction. For this reason, we compare the first non-zero natural frequency for both boundary condition cases.

Indeed, the pinned-pinned thorax has a first non-zero natural frequency of 348 $\mathrm{Hz}$, which is $67 \mathrm{~Hz}$ higher than the free-free thorax natural frequency of $281 \mathrm{~Hz}$. 
This suggests that the experimental boundary conditions may increase the measured the thorax natural frequency. Increasing the clamped area tends to increase the natural frequencies even further. Moreover, the vibration mode shape itself is altered. Whereas the first vibration mode of the free-free thorax is symmetric about the $x$ axis, this symmetry is broken for the pinned-pinned case. Given these limitations of the experimental set-up, future efforts will be devoted to repeating portions of this experiment with boundary conditions more representative of free flight. Nonetheless, the data collected here supports the notion that many insects with asynchronous flight muscles flap at the fundamental frequency of their thorax.

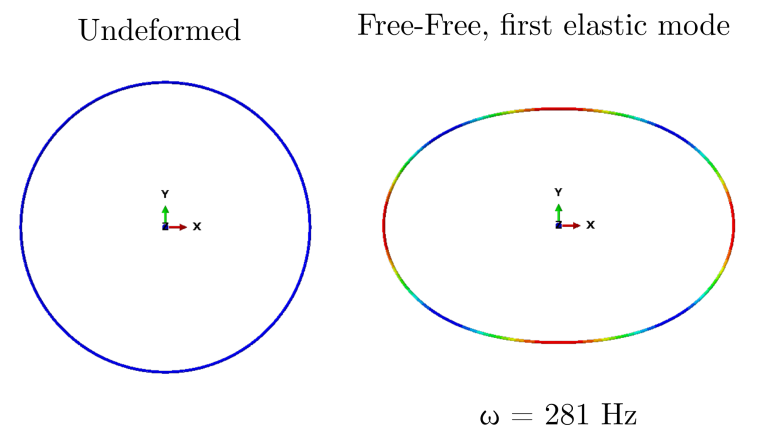

Pinned-Pinned, first mode

Figure 8: Simplified FEA model to demonstrate the effect of boundary condition on the insect thorax frequency response. The pinned-pinned model has a first natural frequency of $67 \mathrm{~Hz}$ higher than the free-free model.

\section{References}

[1] Z Jane Wang. The role of drag in insect hovering. Journal of Experimental Biology, 207(23):41474155, 2004.

[2] Steven N Fry, Rosalyn Sayaman, and Michael H Dickinson. The aerodynamics of free-flight maneuvers in drosophila. Science, 300(5618):495-498, 2003.

[3] Sujay Balebail, Sathish K Raja, and Sanjay P Sane. Landing maneuvers of houseflies on vertical and inverted surfaces. PloS one, 14(8):e0219861, 2019.

[4] Robert J Wood. The first takeoff of a biologically inspired at-scale robotic insect. Robotics, IEEE Transactions on, 24(2):341-347, 2008.

[5] Rajkiran Madangopal, Zaeem Ashraf Khan, and Sunil K Agrawal. Energetics-based design of small flapping-wing micro air vehicles. IEEE/ASME Transactions on Mechatronics, 11(4):433-438, 2006.

[6] Robert C Michelson and Steven Reece. Update on flapping wing micro air vehicle research-ongoing work to develop a flapping wing, crawling entomopter. In 13th Bristol International RPV/UAV Systems Conference Proceedings, Bristol England, volume 30, pages 30-1, 1998.

[7] JK Shang, Stacey A Combes, BM Finio, and Robert J Wood. Artificial insect wings of diverse morphology for flapping-wing micro air vehicles. Bioinspiration $\mathcal{E}$ biomimetics, 4(3):036002, 2009.

[8] Gih-Keong Lau, Yao-Wei Chin, Joel Tian-Wei Goh, and Robert J Wood. Dipteran-insect-inspired thoracic mechanism with nonlinear stiffness to save inertial power of flapping-wing flight. IEEE Transactions on Robotics, 30(5):1187-1197, 2014. 
[9] Sawyer B Fuller, Michael Karpelson, Andrea Censi, Kevin Y Ma, and Robert J Wood. Controlling free flight of a robotic fly using an onboard vision sensor inspired by insect ocelli. Journal of The Royal Society Interface, 11(97):20140281, 2014.

[10] Adam Jaroszewicz, Krzysztof Sibilski, Anna Sibilska, and Andrzej Zyluk. Biomimic sensors guided flight stability and control for flapping wings autonomous micro air vehicles (entomopter). In 45th AIAA Aerospace Sciences Meeting and Exhibit, page 664, 2007.

[11] Hoang Vu Phan and Hoon Cheol Park. Insect-inspired, tailless, hover-capable flapping-wing robots: Recent progress, challenges, and future directions. Progress in Aerospace Sciences, page 100573, 2019.

[12] Michael H Dickinson and Michael S Tu. The function of dipteran flight muscle. Comparative Biochemistry and Physiology Part A: Physiology, 116(3):223-238, 1997.

[13] A Roland Ennos. A comparative study of the flight mechanism of diptera. Journal of Experimental Biology, 127(1):355-372, 1987.

[14] JWS Pringle. The excitation and contraction of the flight muscles of insects. The Journal of physiology, 108(2):226-232, 1949.

[15] Jeff Gau, Nick Gravish, and Simon Sponberg. Indirect actuation reduces flight power requirements in manduca sexta via elastic energy exchange. Journal of the Royal Society Interface, 16(161):20190543, 2019.

[16] Robert K Josephson, Jean G Malamud, and Darrell R Stokes. Asynchronous muscle: a primer. Journal of Experimental Biology, 203(18):2713-2722, 2000.

[17] FW Darwin and John William Sutton Pringle. The physiology of insect fibrillar muscle-i. anatomy and innervation of the basalar muscle of lamellicorn beetles. Proceedings of the Royal Society of London. Series B. Biological Sciences, 151(943):194-203, 1959.

[18] KE Machin and John William Sutton Pringle. The physiology of insect fibrillar muscle-ii mechanical properties of a beetle flight muscle. Proceedings of the Royal Society of London. Series B. Biological Sciences, 151(943):204-225, 1959.

[19] KE Machin and John William Sutton Pringle. The physiology of insect fibrillar muscle iii. the effect of sinusoidal changes of length on a beetle flight muscle. Proceedings of the Royal Society of London. Series B. Biological Sciences, 152(948):311-330, 1960.

[20] KE Machin, John William Sutton Pringle, and M Tamasige. The physiology of insect fibrillar muscle-iv. the effect of temperature on a beetle flight muscle. Proceedings of the Royal Society of London. Series B. Biological Sciences, 155(961):493-499, 1962.

[21] R Dudley and CP Ellington. Mechanics of forward flight in bumblebees: I. kinematics and morphology. Journal of Experimental Biology, 148(1):19-52, 1990.

[22] Fritz-Olaf Lehmann and Michael H Dickinson. The production of elevated flight force compromises manoeuvrability in the fruit fly drosophila melanogaster. Journal of Experimental Biology, 204(4):627-635, 2001.

[23] JA Miyan and Arthur W Ewing. How diptera move their wings: a re-examination of the wing base articulation and muscle systems concerned with flight. Philosophical Transactions of the Royal Society of London. B, Biological Sciences, 311(1150):271-302, 1985.

[24] John William Sutton Pringle. Insect flight, volume 9. Cambridge University Press, 1957.

[25] S Sponberg and TL Daniel. Abdicating power for control: a precision timing strategy to modulate function of flight power muscles. Proceedings of the Royal Society of London B: Biological Sciences, page rspb20121085, 2012.

[26] Douglas L Altshuler, William B Dickson, Jason T Vance, Stephen P Roberts, and Michael H Dickinson. Short-amplitude high-frequency wing strokes determine the aerodynamics of honeybee flight. Proceedings of the National Academy of Sciences, 102(50):18213-18218, 2005.

[27] Andrew M Mountcastle and Stacey A Combes. Wing flexibility enhances load-lifting capacity in bumblebees. Proceedings of the Royal Society B: Biological Sciences, 280(1759):20130531, 2013.

[28] Michael Hrncir, Anne-Isabelle Gravel, Dirk Louis P Schorkopf, Veronika M Schmidt, Ronaldo Zucchi, and Friedrich G Barth. Thoracic vibrations in stingless bees (melipona seminigra): 
resonances of the thorax influence vibrations associated with flight but not those associated with sound production. Journal of Experimental Biology, 211(5):678-685, 2008.

[29] Noriyasu Ando and Ryohei Kanzaki. Flexibility and control of thorax deformation during hawkmoth flight. Biology letters, 12(1):20150733, 2016.

[30] Michael E Schauff. Collecting and preserving insects and mites: techniques and tools. Systematic Entomology Laboratory, USDA, 2001.

[31] Aaron G Norris, Anthony N Palazotto, and Richard G Cobb. Experimental structural dynamic characterization of the hawkmoth (manduca sexta) forewing. International Journal of Micro Air Vehicles, 5(1):39-54, 2013.

[32] Robert E Snodgrass. Anatomy of the honey bee. Cornell University Press, 1956.

[33] Paul A De Luca and Mario Vallejo-Marín. What's the buzzabout? the ecology and evolutionary significance of buzz-pollination. Current opinion in plant biology, 16(4):429-435, 2013.

[34] Sarah A Corbet and Shuang-Quan Huang. Buzz pollination in eight bumblebee-pollinated pedicularis species: does it involve vibration-induced triboelectric charging of pollen grains? Annals of botany, 114(8):1665-1674, 2014.

[35] Marcus J King, Stephen L Buchmann, and Hayward Spangler. Activity of asynchronous flight muscle from two bee families during sonication (buzzing). Journal of Experimental Biology, 199(10):2317-2321, 1996.

[36] Ivana Kovacic and Michael J Brennan. The Duffing equation: nonlinear oscillators and their behaviour. John Wiley \& Sons, 2011.

[37] Fareed Kaviani and Hamid Reza Mirdamadi. Snap-through and bifurcation of nano-arches on elastic foundation by the strain gradient and nonlocal theories. International Journal of Structural Stability and Dynamics, 13(05):1350022, 2013.

[38] Jan Michels, Esther Appel, and Stanislav N Gorb. Functional diversity of resilin in arthropoda. Beilstein journal of nanotechnology, 7(1):1241-1259, 2016.

[39] Svend Olav Andersen and Torkel Weis-Fogh. Resilin. a rubberlike protein in arthropod cuticle. In Advances in insect physiology, volume 2, pages 1-65. Elsevier, 1964.

[40] R Josephson and C Ellington. Power output from a flight muscle of the bumblebee bombus terrestris. i. some features of the dorso-ventral flight muscle. Journal of Experimental Biology, 200(8):1215-1226, 1997.

[41] Bo Cheng, Xinyan Deng, and Tyson L Hedrick. The mechanics and control of pitching manoeuvres in a freely flying hawkmoth (manduca sexta). Journal of Experimental Biology, 214(24):40924106, 2011.

[42] Heidi E Reid, Ryan K Schwab, Miles Maxcer, Robert KD Peterson, Erick L Johnson, and Mark Jankauski. Wing flexibility reduces the energetic requirements of insect flight. Bioinspiration 83 biomimetics, 14(5):056007, 2019.

[43] Marcos Vanella, Timothy Fitzgerald, Sergio Preidikman, Elias Balaras, and Balakumar Balachandran. Influence of flexibility on the aerodynamic performance of a hovering wing. Journal of Experimental Biology, 212(1):95-105, 2009.

[44] Mark Jankauski, Ziwen Guo, and IY Shen. The effect of structural deformation on flapping wing energetics. Journal of Sound and Vibration, 429:176-192, 2018.

[45] Jason T Vance, Douglas L Altshuler, William B Dickson, Michael H Dickinson, and Stephen P Roberts. Hovering flight in the honeybee apis mellifera: kinematic mechanisms for varying aerodynamic forces. Physiological and Biochemical Zoology, 87(6):870-881, 2014.

[46] Michael E Dillon and Robert Dudley. Surpassing mt. everest: extreme flight performance of alpine bumble-bees. Biology letters, 10(2):20130922, 2014.

[47] Stephen P Roberts, Jon F Harrison, and Robert Dudley. Allometry of kinematics and energetics in carpenter bees (xylocopa varipuncta) hovering in variable-density gases. Journal of Experimental Biology, 207(6):993-1004, 2004.

[48] Jason T Vance and Stephen P Roberts. The effects of artificial wing wear on the flight capacity of the honey bee apis mellifera. Journal of insect physiology, 65:27-36, 2014. 
[49] DM Unwin and Sarah A Corbet. Wingbeat frequency, temperature and body size in bees and flies. Physiological Entomology, 9(1):115-121, 1984.

[50] Kim Billy Blair, CM Krousgrill, and TN Farris. Non-linear dynamic response of shallow arches to harmonic forcing. Journal of sound and vibration, 194(3):353-367, 1996. 